Anales de Geografía de la Universidad Complutense ISSN: 0211-9803

http://dx.doi.org/10.5209/AGUC.64676

\title{
Estimación de la subida del nivel del mar por efecto del Cambio Climático mediante datos de mareógrafos y la serie de satélie NASA Topex-Poseidon/Jason. El caso de Valencia
}

\author{
Touria Dawahidi ${ }^{1}$; Dadiel Ibarra Marinas ${ }^{2}$; Francisco Gomariz-Castillo ${ }^{3}$ \\ Recibido: 15 de marzo del 2018/ Enviado a evaluar: 19 de octubre del 2018/ Aceptado: 27 de febrero del 2019
}

Resumen. La subida del nivel del mar es una de las consecuencias más relevantes derivado del Cambio Climático. Los estudios relacionados con la subida del nivel del mar muestran una gran variabilidad espacial. Este trabajo se ha centrado en el área litoral de la ciudad de Valencia, situada en el Mediterráneo de la Península Ibérica. La proyección de la subida del nivel del mar se ha estimado a partir de altimetrías multimisión de satélite y del Commonwealth Scientific and Industrial Research Organisation para los escenarios Representative Concentration Pathway RCP2.6, RCP4.5 y RCP8.5, regionalizados mediante regresión lineal y los registros históricos de los mareógrafos del Permanent Servicefor Mean Sea Level. Los resultados muestran incrementos entre 27,59 y 143,63 cm, (R2=0,62 para mareógrafos y $\mathrm{R} 2=0,37$ para satélites), para finales del S.XXI. Las consecuencias implican la intensificación del efecto de los temporales marítimos y el aumento de la vulnerabilidad de las áreas costeras.

Palabras clave: Vulnerabilidad costera; cambio climático; subida del nivel del mar; altimetría de satélite.

[en] Accuracy measurement of sea level rise due to effect of Climate Change through tide gauge data and NASA Topex-Poseidon/Jason satellite series. The case of Valencia

Abstract. The rise in sea level is one of the most important consequences of Climate Change. Studies related to the sea level rise show great spatial variability. This study focused on the coastal area of Valencia, located in the Mediterranean of the Iberian Peninsula. The projection of the rise in sea level has been estimated from satellite multisite altimetry and Commonwealth Scientific and Industrial Research Organisation data for the Representative Concentration Pathway scenarios RCP2.6, RCP4.5 and RCP8.5, regionalized by a linear regression model and historical records of the tide gauges of the Permanent Service for Mean Sea Level. The results show increases in sea levels from 27.59 and 143,63

1 Departamento de Geografía. Universidad de Murcia.

E-mail: touria.dawahhidi@um.es

2 Departamento de Geografia. Universidad de Murcia.

E-mail: adaniel.ibarra@um.es

3 Departamento de Geografía. Universidad de Murcia. Instituto Euromediterráneo del Agua.

E-mail: fjomariz@um.es 
$\mathrm{cm},(\mathrm{R} 2=0.62$ from tide gauges and $\mathrm{R} 2=0.37$ from satellites data), by the end of the 21 st century. Possible consequences include the intensification of the effect of maritime storms and an increased vulnerability of the coastal areas.

Keywords: Coastal vulnerability; Climate Change; sea level rise; satellite altimetry.

[fr] Estimation de la montée du niveau de la mer par effet du Changement Climatique selon les donnés des marégraphes et la série de satellites NASA Topex-Poseidon/Jason. Le cas de Valence

Résumé. L'élévation du niveau de la mer est l'une des conséquences les plus importantes du changement climatique. Les études relatives à l'élévation du niveau de la mer montrent une grande variabilité spatiale. Cette étude s'est concentrée sur la zone côtière de Valence, située dans la Méditerranée de la péninsule ibérique. La projection de l'élévation du niveau de la mer a été estimée à partir des données altimétriques multisites satellitaires et des données de l'Organisation de recherche scientifique et industrielle du Commonwealth pour les scénarios RCP2.6, RCP4.5 et RCP8.5, régionalisés par un modèle de régression linéaire et les enregistrements historiques des marégraphes du Service permanent du niveau moyen de la mer. Les résultats montrent une élévation du niveau de la mer de 27,59 et 143,63 cm (R2 =0,62 d'après les marégraphes et $\mathrm{R} 2=0,37$ d'après les données satellitaires), d'ici la fin du 21e siècle. Parmi les conséquences possibles figurent l'intensification de l'effet des tempêtes maritimes et une vulnérabilité accrue des zones côtières.

Mots clés: Vulnérabilité côtière; Changement Climatique; élévation du niveau de la mer; altimétrie satellitaire.

Cómo citar. Dawahidi, T., Ibarra Marinas, D. y Gomariz-Castillo, F. (2019): Estimación de la subida del nivel del mar por efecto del Cambio Climático mediante datos de mareógrafos y la serie de satélie NASA Topex-Poseidon/Jason. El caso de Valencia. Anales de Geografia de la Universidad Complutense, 39(1), 39-58.

Sumario. 1. Introducción. 2. Materiales y métodos. 2.1. Área de estudio. 2.2. Fuentes y procesamiento de la información. 2.3. Regionalización estadística y predicción de las series de LMSL (Local Mean Sea Level). 3. Resultados y discusión. 4. Conclusiones. 5. Referencias bibliográficas.

\section{Introducción}

El nivel medio del mar (Mean Sea Level - MSL) se define como el nivel de las aguas tranquilas del mar promediado durante un periodo determinado de tiempo (meses, años) de tal forma que los efectos provocados periódicamente por mareas y por otras causas frecuentes como las olas queden compensados (Pons Valls, 2009); por esta y otras razones, la evolución del nivel medio del mar es una variable fundamental que explica numerosos procesos físicos de la Tierra (Fraile Jurado y Ojeda Zújar, 2012).

El planeta ha experimentado considerables modificaciones antrópicas desde el siglo XVIII hasta la actualidad (Lizano y Lizano, 2010). El Cambio Climático es consecuencia directa de estos procesos y constituye uno de los grandes desafíos a lo que se enfrenta la sociedad presente y futura (Hidalgo y Picaso, 2010).

El Cambio Climático, producido principalmente por el aumento de las emisiones de Gases de Efecto Invernadero (GEI) generadas por la actividad humana, es un 
fenómeno que ha aumentado recientemente debido entre otros factores a la explotación de los combustibles fósiles, petróleo y carbón. El consumo de estos fósiles ha provocado un incremento de los gases más contribuyentes al calentamiento global, el CO2, que registra entre 1970 y 2004 un aumento del $80 \%$, pasando de 21 a 30 Gigatoneladas (García Fernández, 2010).Las evidencias muestran que el Cambio Climático está causado principalmente por las actividades humanas, que han generado un aumento de la temperatura de la atmósfera terrestre. Algunos autores confirman que de los doce últimos años del periodo 1995-2006, once se encuentran entre los más calurosos, desde que se inició la elaboración de los registros instrumentales de temperatura de la superficie del planeta en 1850 (Pernía Llera y Fornés Azcoiti, 2008). Aunque el aumento de las temperaturas se halla distribuido de forma generalizada por el planeta, existen sectores más vulnerables, como las áreas septentrionales, en especial en la región Ártica. También se acentúan más en las regiones terrestres que en la oceánicas (IPCC, 2007). Las proyecciones futuras predicen aumentos de hasta $4^{\circ} \mathrm{C}$ antes del periodo 2055 (IPCC, 2014).

Uno de los indicadores más significativos de este cambio es el aumento del nivel del mar, uno de los principales retos a los que la sociedad se enfrenta en la actualidad (IPCC, 2014) y que constituye un problema creciente, debido a su aceleración a lo largo de la última década (Yi et al., 2016). A largo plazo han existido cambios en el nivel del mar lentos y cíclicos, debidos a parámetros orbitales de 900 años de periodo (Loutre et al., 1992), oscilaciones del sistema formado por el océano- atmósfera con ciclos en torno a los 1.500 años (Bond et al., 2013) y fluctuaciones en la circulación termohalina que pueden provocar ciclos de periodos que varían entre 550 y 1.000 años (Chapman y Shackleton, 2000).

Durante el último período interglaciar cálido el nivel del mar se encontraba entre 6 y $8 \mathrm{~m}$ por encima del nivel actual y durante el último período glacial, aproximadamente $130 \mathrm{~m}$ más bajo. A pesar de la gran variabilidad que muestran los registros geológicos, el nivel del mar ha sido bastante estable durante los últimos 3.000 años anteriores a la segunda mitad del siglo XIX (Williams, 2013). Antes de 1990, el nivel de la media global se encontraba en 1,1 mm/año, sin embargo de 1993 a 2012 el aumento medio se triplicó (Dangendorfet et al., 2017) pasando a 3,1 $\mathrm{mm} / \mathrm{año}$. En la actualidad este fenómeno se debe principalmente al derretimiento glacial de Groenlandia y la Antártida, a la expansión térmica oceánica, ligada al aumento de la temperatura de los océanos, y a una caída en la contribución de nieve y permafrost sobre la tierra. Durante el periodo 1993-2007, la contribución del deshielo continental ha sido del $55 \%$, pero a partir de 2007 , el derretimiento de las masas de hielo continental ha pasado a representar el 80\% (Cazenave y Llowell, 2010), debido a la aceleración del deshielo.

El incremento del nivel medio del mar debido al calentamiento de los océanos y las pérdidas de las masas de hielo, causará graves problemas en las áreas costeras, consideradas uno de los ambientes más frágiles del medio terrestre (Ibarra Marinas et al., 2015), como pérdidas de tierra habitable y cultivable, intrusión de agua salada y erosión costera (Ibarra Marinas, 2016), situación que afecta al 70\% de las playas del 
planeta (Arteaga Cardineau, 2007-2008; Tavares Correa y Drenkhan, 2010). Por ello, las instituciones encargadas de realizar las estrategias y planes para identificar, valorar y prevenir los efectos de la subida del nivel del mar, recomiendan la elaboración de estudios globales y regionales con el fin de determinar, a gran escala, las consecuencias de las subida del nivel del mar (Fraile Jurado et al., 2014). Además, la aplicación, en leyes urbanísticas y de planificación territorial costera, de umbrales que delimiten posibles territorios que pueden verse afectados por la subida del nivel del mar en un futuro (Schubert et al., 2006), ya que se estima que el $60 \%$ de habitantes del conjunto del planeta se localizan en menos de $100 \mathrm{~km}$ de la costa, desarrollo que se intensificara por el proceso de litoralización que se está difundiendo actualmente (Del Rio et al., 2015).

Los registros a largo plazo de las estaciones meteorológicas en la Península Antártica muestran fuertes tendencias de aumento de las condiciones de derretimiento del hielo continental (Vaughan, 2006). Los efectos de la subida del nivel del mar varían según la tipología de las costas. Mientras en las costas acantiladas las consecuencias son insignificantes, los deltas, humedales y playas urbanas presentan una gran vulnerabilidad. Entre las consecuencias más visibles se encuentran la erosión costera, las inundaciones por temporales (Benavente et al., 2006) y los daños ambientales relacionados.

En un clima cambiante, el aumento del nivel global del mar, tendrá cambios en los patrones en la trayectoria de las tormentas y tempestades, este hecho unido al aumento de la densidad de población y las presiones sobre la zona costera, supone el aumento del futuros riesgos de impactos de las tormentas costeras (Brown et al., 2016). En este sentido, tanto desde un punto de vista natural como socioeconómico, las costas del litoral mediterráneo, suponen una de las áreas más vulnerables, por lo que se hacen cada vez más necesarios estudios que evalúen los impactos producidos en la costa.

Pese a que los primeros estudios de nivel del mar se inician partir de la segunda mitad del siglo XIX, es a partir de la década de 1930, cuando se desarrollan sistemas de referencia capaces de aportar datos históricos locales. La fundación de un organismo internacional responsable de la recogida, el almacenamiento y la publicación de los datos del nivel del mar de los mareógrafos de todo el mundo, el Permanent Service for Mean Sea Level (PSMSL) en 1933, facilitó la difusión de las altimetrías a nivel global. A partir de mediados del siglo XX, los cambios de nivel marino, se integran como una de las variables de la erosión costera (Bruun, 1962), debido a su implicación en el retroceso de los perfiles costeros. A partir de la década de 1990, los trabajos realizados a partir de series temporales (Spencer y Woodworth, 1993) han mostrado una tendencia a la aceleración de este proceso (Nerem, 1999). En la actualidad, el aumento en la disponibilidad de datos ha encaminado los esfuerzos a la modelización de nuevos escenarios (Church y White, 2011; Jevrejeva et al., 2012; Álvarez Francoso et al., 2017). Los estudios relacionados con los cambios en el nivel del mar en el litoral español, por efecto del Cambio Climático comienzan a ser relativamente frecuentes a partir de los últimos años de la década de 1990 (Ibañez et al., 1996; González y Medina, 2001). En la actualidad son comunes los trabajos que 
relacionan este fenómeno con los efectos sobre las playas y la vulnerabilidad costera a este fenómeno (Ojeda-Zujar et al., 2009; Alvarado-Aguilar et al., 2012) y las proyecciones a partir forzamientos climáticos (Jevrejeva, et al., 2016). En los últimos años en España, cabe destacar el estudio realizado por Fraile Jurado (2011), donde se aborda tanto la metodología en la representación espacial como en la evaluación de riesgos asociados al incremento del nivel del mar.

Actualmente, la subida del nivel del mar genera una fuerte discusión científica, se ha concentrado en el cálculo global de este aspecto, utilizando frecuentemente el horizonte 2100, aunque existen trabajos que indican que el nivel continuará ascendiendo durante varios siglos (Jevrejeva et al., 2012). Las predicciones del nivel del mar, desde el punto de partida de los modelos predictivos hasta el día de hoy, generó debates entre los modelos propuesto por el Grupo Intergubernamental de Expertos sobre el Cambio Climático (Intergovernmental Panel on Climate Change IPCC) y los expresados por otros autores, según estos últimos los datos del IPCC conceden valores inferiores de la subida real (Fraile Jurado et al., 2014). Estas discusiones han generado la realización de diversos estudios con estimaciones de la subidas del nivel del mar de hasta 2 metros a finales del siglo XXI (Rahmstorf, 2007) estimación variante en función del área de estudio. Mengel et al. (2018) estiman un aumento medio global del nivel del mar entre 0,7 y $1,2 \mathrm{~m}$, en el caso más optimista de emisiones de gases de efecto invernadero netas nulas, llegando a la conclusión de que la estabilización de las temperaturas por debajo de $2{ }^{\circ} \mathrm{C}$ propuesta en la cumbre de París es insuficiente. Este problema se ve acentuado por la elevada inercia y baja reversibilidad del aumento del nivel del mar, incluso si se cumplen los acuerdos de París, pudiendo continuar en ascenso hasta el año 2300.

El objetivo de este trabajo ha sido la estimación de la subida del nivel del mar en la ciudad de Valencia y su entorno a partir de los datos procedentes de elevaciones de satélite y de las series procedentes de Church y White (2011), que dispone de información de elevaciones para tres escenarios de cambio climático, para el periodo 2080-2100. La regionalización estadística ha permitido estimar el nivel medio local del mar ajustado a las características locales del área de estudio.

\section{Materiales y métodos}

En los siguientes apartados se describe el área de estudio, fuentes de información y método de regionalización estadística para obtener las series finales. Para ello se propone un método sencillo de estimación basado en regresiones lineales entre las series globales del nivel del mar (Global Mean Sea Level-GMSL) y las series históricas de elevación media local (Local Mean Sea Level-LMSL) en el área de estudio, obteniendo como resultado proyecciones de éstas en los escenarios de cambio climático considerados.

El proceso de trabajo se ha realizado a partir de los programas de código abierto Quantum GIS (QGIS Development Team, 2017) para el manejo de información 
espacial y R-CRAN (R Core Team, 2017) para la adquisición, depuración y análisis de los datos de forma automatizada. El uso de este tipo de herramientas ha permitido automatizar desde la descarga de la información hasta su análisis y obtención de los resultados, permitiendo la reproducibilidad del estudio a otras áreas de trabajo y series de datos de forma sencilla.

\section{1. Área de estudio}

El área de estudio incluye el Área Metropolitana de Valencia y el norte de L'Albufera (Figura 1). La costa de la Provincia de Valencia se extiende en dirección N-S a lo largo de $109 \mathrm{Km}$ en el Mediterráneo, de los cuales13Km pertenecen a la capital. El litoral presenta una morfología de costa baja caracterizado por la presencia de formaciones de restinga-albufera y áreas fácilmente inundables (Sanjaumeet et al., 1996).

Los fondos marinos son mayoritariamente arenosos y forman una batimetría sin grandes irregularidades. La dirección de la deriva litoral es N-S y la altura de ola significante no suele superar el metro. Como en la mayor parte del litoral mediterráneo español, de carácter micromareal, la carrera de marea es escasa.

Figura 1. Área Metropolitana de Valencia. Playas urbanas y Albufera de Valencia.

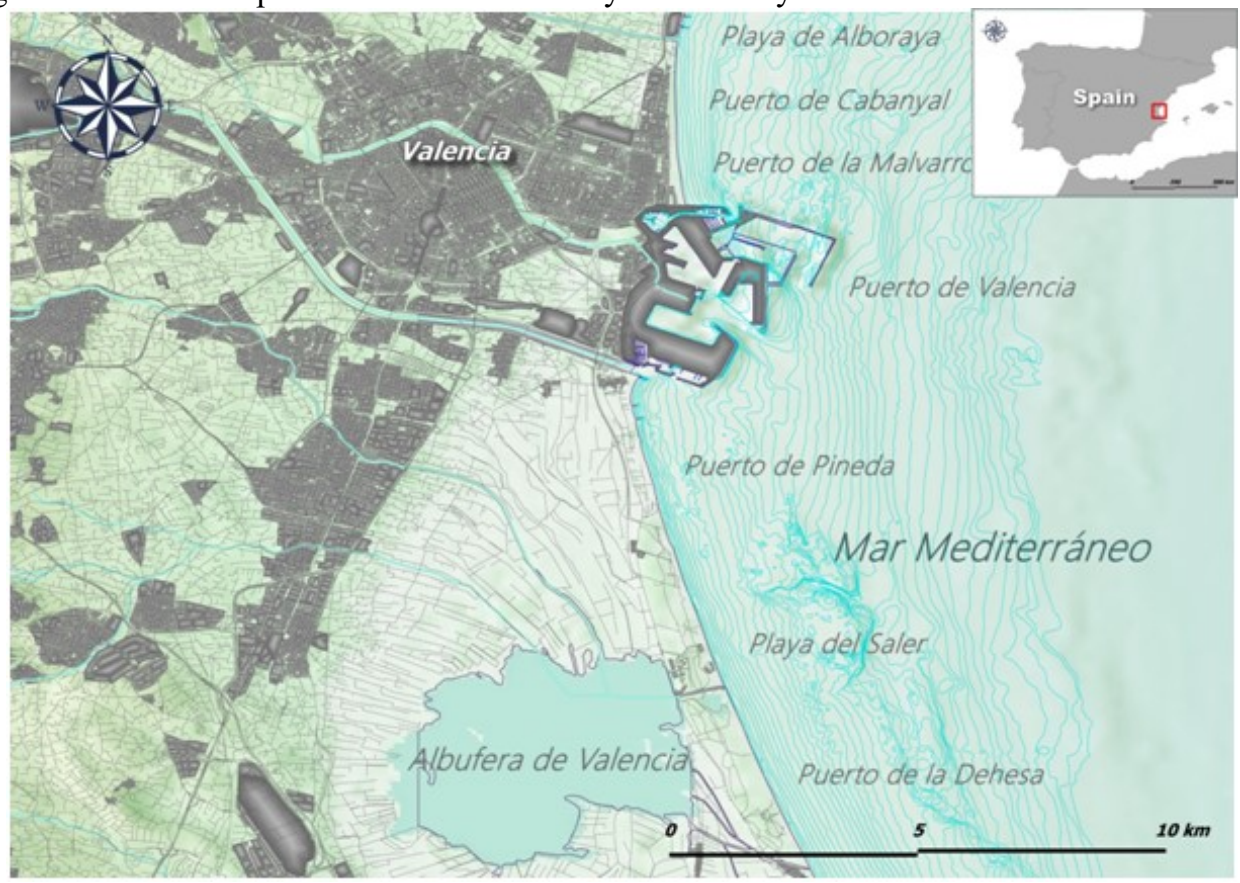

Fuente: Elaboración propia, a partir de datos de Cartociudad (IGN, 2017), las Ecocartografías de Valencia (MAPAMA, 2017) y los datos del Marine Information Service (2016). 
A pesar de que el aumento de las edificaciones próximas a la costa en los últimos años, ha sido menor que en las áreas turísticas de la Comunidad Valenciana, gran parte de la población se concentra en la capital (Pitarch y Membrado, 2014).

No obstante, resulta difícil predecir los procesos de urbanización que se llevarán a cabo hasta el periodo (2080-2100) y que pueden estar afectados por los temporales marinos presentes en la zona en especial en los meses de noviembre, diciembre y enero, como muestran las alturas medias y máximas mensuales del modelo de reanálisis SIMAR para el periodo 2006-2018 (Figura 2).

Figura 2. Altura de ola significante. Medias y máximos mensuales.

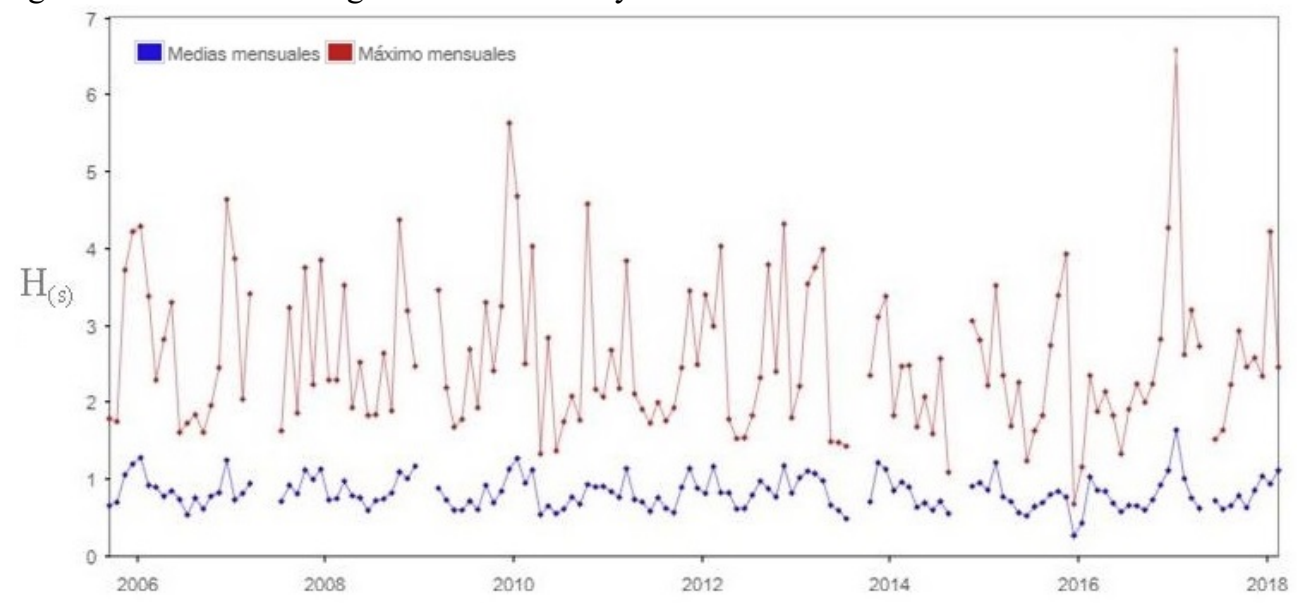

Fuente: Puertos del Estado (2017).

Las costas de restinga ocupan más de la mitad de la costa de la Comunidad Valenciana (Eguibar Galanet et al., 2007), hecho que aumenta la vulnerabilidad de esta comunidad a los cambios de nivel marítimos. Otra de las áreas más vulnerables a los cambios en el nivel del mar son las playas urbanas de la ciudad de Valencia, como las de Cabanyal y la Malvarrosa. Adicionalmente, se han considerado otras playas del municipio: las playas de Mediana y Alborada-Port Sa Playa, situadas al norte de la ciudad, y las de Pinedo, Saler y Dehesa, situadas al sur.

\subsection{Fuentes y procesamiento de la información}

Las proyecciones de altura media del nivel del mar se han realizado a partir de las series globales de datos altimétricos derivados de los satélites TOPEX-Poseidon y JASON, que permiten la modelización tanto de tendencias globales como regionales (Neremet et al., 2018). Estas series presentan datos de altura media con una resolución respecto a la superficie del geoide de 1 grado y una precisión vertical máxima de $0,15 \mathrm{~mm}$. La selección de los puntos de satélite se ha realizado de forma aleatoria y a una distancia mínima de 1 grado de la costa, con el objetivo de evitar el 
ruido generado por las celdillas de tipo pixel mixto, en las que comparten valores la superficie terrestre y oceánica (Fraile Jurado, 2011).

Además de los datos altimétricos, para la construcción de la serie global se han empleado las series de datos del Commonwealth Scientific and Industrial Research Organisation (CSIRO), obtenidas a partir de 250 mareógrafos (Figura 3). Los datos corresponden al aumento del nivel medio del mar en el mundo a partir de las medias de los datos de altímetros de satélites para el periodo 1993-2009 (Church y White, 2011). Esta reconstrucción, que corrige el ajuste isostático glacial, utiliza datos mensuales procedentes de los mareógrafos del Permanent Service for Mean Sea Level (Holgateet et al., 2013), y datos procedentes de TOPEX-Poseidon/ JASON, para reconstruir curvas GMSL desde 1870 a 2009 mediante funciones empíricas ortogonales (Empirical Orthogonal Functions - EOF). El uso de esta serie permite adicionalmente caracterizar la evolución temporal del GMSL.

Para el ajuste de las series globales a la zona de estudio se ha utilizado la serie histórica procedente de los mareógrafos del PSML. La ventaja principal de éstos datos sobre los procedentes de los satélites es que su tratamiento permite la obtención de las tendencias pasadas, teniendo en cuenta los cambios por el efecto de la subsidencia (Fraile Jurado et al., 2014).

Figura 3. Serie temporal del nivel medio del mar global SCIRO (1993-2009).

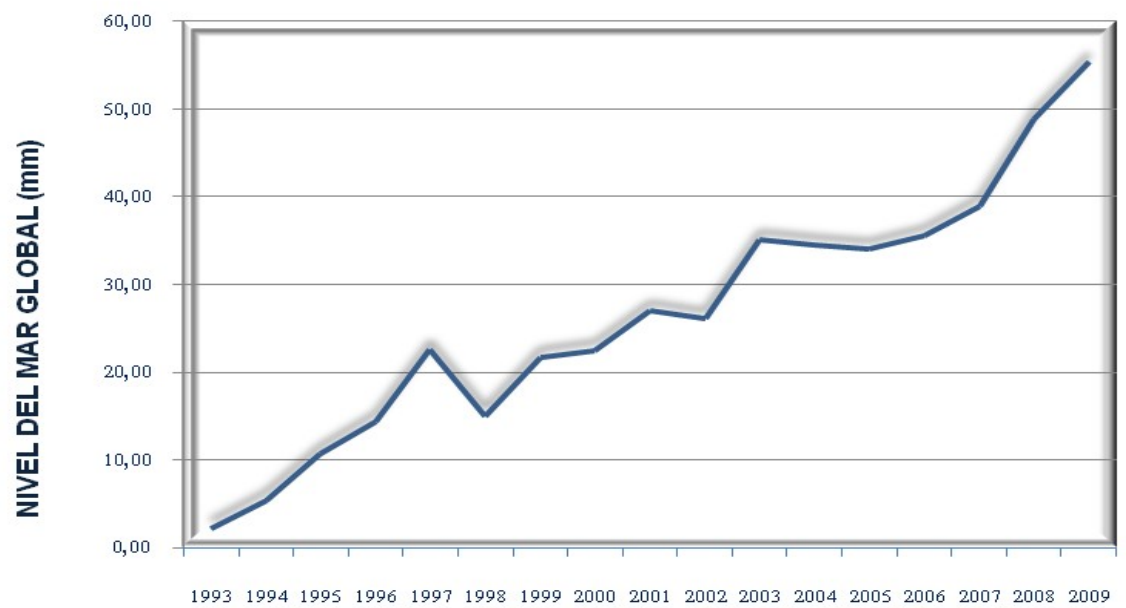

Fuente: Elaboración propia, a partir de los datos de Church y White (2011).

Para evaluar el efecto del cambio climático se han utilizado tres escenarios, definidos por las Trayectorias o Sendas Representativas de Concentración de gases de efecto invernadero (Representative Concentration Pathway - RCP) del Quinto Informe de Evaluación del IPCC (Fifth Assessment Report - AR5), para el periodo 2080-2100. Los RCP son escenarios caracterizados por el Forzamiento Radiativo total al que se ven sometidos para el año 2100 respecto a 1750, en función de las emisiones 
debido a factores antropogénicos (combinaciones de posibles futuros económicos, tecnológicos, demográficos y políticos) y los esfuerzos de mitigación derivados de las diversas políticas. El rango de Forzamiento Radiativo oscila entre 2,6 W/m (escenario de forzamiento muy bajo o de mitigación RCP2.6) y $8,5 \mathrm{~W} / \mathrm{m}^{2}$ (escenario de forzamiento muy elevado RCP8.5). En base a estos escenarios se realizan proyecciones mediante modelización, estimando sus efectos a nivel global.

En este estudio se han utilizado tres escenarios: a) RCP2.6 (escenario de mitigación), que presenta una subida del nivel del mar global de 0,40 m (Van Vuuren et al., 2007); b) RCP4.5 (emisiones intermedias o escenario de estabilización), donde se estima una proyección de subida global de $0,47 \mathrm{~m}$ (Clarke et al., 2007); c) RCP8.5 (altas emisiones), que representa las subidas a nivel global más extremas de $0,63 \mathrm{~m}$ (Riahiet et al., 2007). No se ha considerado el escenario RCP6.0, ya que las proyecciones de aumento del nivel del mar para el siglo XXI son similares a las del RCP 4.5. Los cuatro RCP se basan en escenarios de emisión de gases múltiples (Meinshausen et al., 2011; Wise et al. 2009), cuya metodología se desarrolla en detalle en IPCC (2016).

\subsection{Regionalización estadística y predicción de las series de LMSL (Local Mean Sea Level)}

Para generar las proyecciones regionalizadas de LMSL se han estimado dos modelos de regresión lineal entre los registros recientes de niveles del mar y las series globales de GMSL. Como variables dependientes se han considerado la de Church y White (2011) y como variable independiente la serie local (LMSL). Este tipo de métodos se han utilizado con éxito en estudios como el realizado por Fraile Jurado (2011).

Una vez verificado el modelo mediante hipótesis de linealidad, normalidad, homocedasticidad, no autocorrelación e independencia, mediante los contrastes de Harvey-Collier, Kolmogorov-Smirnov, Durwin-Watson respectivamente, se ha realizado la estimación de los niveles del mar futuros para el periodo 2080-2100 mediante la siguiente ecuación, la cual resume el modelo estimado:

$$
Y_{2080-210}=\beta_{0}+\beta_{1} X+\varepsilon
$$

Siendo: $Y_{2080-210}$ la subida del nivel del mar local en cm, para el periodo 2080-2100, $\beta_{0}$ la constante del modelo, $\beta_{1}$ el coeficiente del downscaling realizado entre la serie global y local, la subida de nivel del mar en m procedente del escenario RCP utilizado, $\varepsilon$ es una variable aleatoria, llamada error, que proviene de una distribución normal con media cero y desviación estándar $\sigma^{2}$.

Del modelo estadístico se obtiene dos rectas de predicción que representan cada uno de los tres escenarios a estimar tanto para la serie del mareógrafo del área de estudio como para los registros procedente del satélite TOPEX-Poseidon/ JASON, y las cuales determinan unos errores asociados a un nivel de confianza del $95 \%$, que 
son comparados con los errores calculados por el IPCC, también asociados al mismo nivel de confianza (IPCC, 2013).

Una vez obtenidas las alturas futuras del nivel del mar para los tres escenarios RCP (40 cm para RCP2.6) $46 \mathrm{~cm}$ para RCP4.5 y $63 \mathrm{~cm}$ para RCP8.5), se ha estimado la subida neta del nivel del mar a partir de la diferencia entre los valores locales de la recta de predicción (LMSL) y la media de los tres últimos años (2007-2009), atendiendo al criterio del patrón observado del cambio de pendiente de la serie de Church y White (2011).

\section{Resultados y discusión}

Los modelos de regresión muestran pendientes positivas y significativas, con pvalores muy inferiores al nivel de significancia $\alpha=0,05$, tanto para los datos procedentes del mareógrafo del Puerto de Valencia (Tabla 1), como para los obtenidos a partir de la serie de satélites (Tabla 2). Los coeficientes de determinación de ambas series presentan diferencias. La serie de los mareógrafos se ajusta mejor, con un $\mathrm{R}^{2}=0,62$, que la serie procedente de los satélites la cual muestra un $\mathrm{R}^{2}=0,37$ (Figuras 5 y 6 ).

Tabla 1. Resumen del modelo estadístico mediante registros del mareógrafo de Valencia.

\begin{tabular}{lrrrr}
\hline \multicolumn{1}{r}{ Parámetro } & Coeficiente & Error Estándar & Estadístico-t & p-valor \\
\hline Constante & $6,99 E+06$ & $6,39 E+03$ & 1094,99 & $<2 e-16^{* * *}$ \\
GMSL & $2,43 E+03$ & $3,13 E+01$ & 77,77 & $<2 e-16^{* * *}$ \\
\hline
\end{tabular}

Fuente: Elaboración propia

Tabla 2. Resumen del modelo estadístico mediante datos de satélite.

\begin{tabular}{lrrrr}
\hline \multicolumn{1}{c}{ Parámetro } & Coeficiente & Error Estándar & Estadístico-t & \multicolumn{1}{c}{ p-valor } \\
\hline Constante & $-2,81 E+04$ & 0.8039 & $-3,49 E+03$ & $0.00327 * *$ \\
GMSL & 0.7900 & 0.2675 & $2,95 E+03$ & $0.00986^{* *}$ \\
\hline
\end{tabular}

Fuente: Elaboración propia. 
Figura 5. Diagrama de dispersión entre la serie global anual y la serie de registros medios anuales del mareógrafo de Valencia.

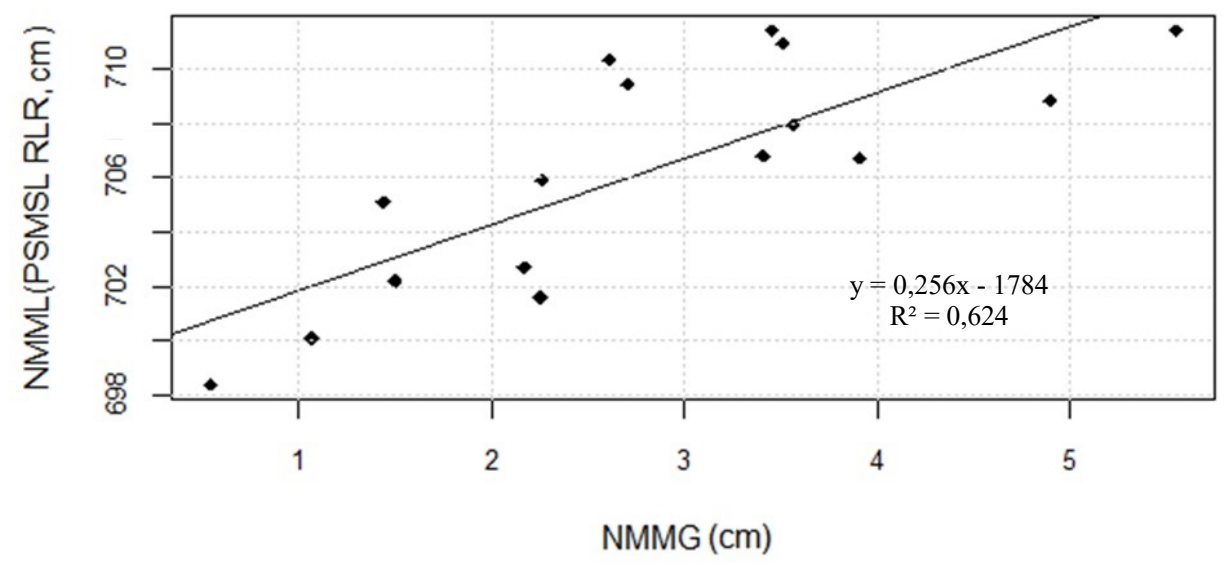

Fuente: Elaboración propia.

Figura 6. Diagrama de dispersión entre la serie global anual y la serie local anual del satélite TOPFX_Pncaidnn/I $\Delta$ CONT

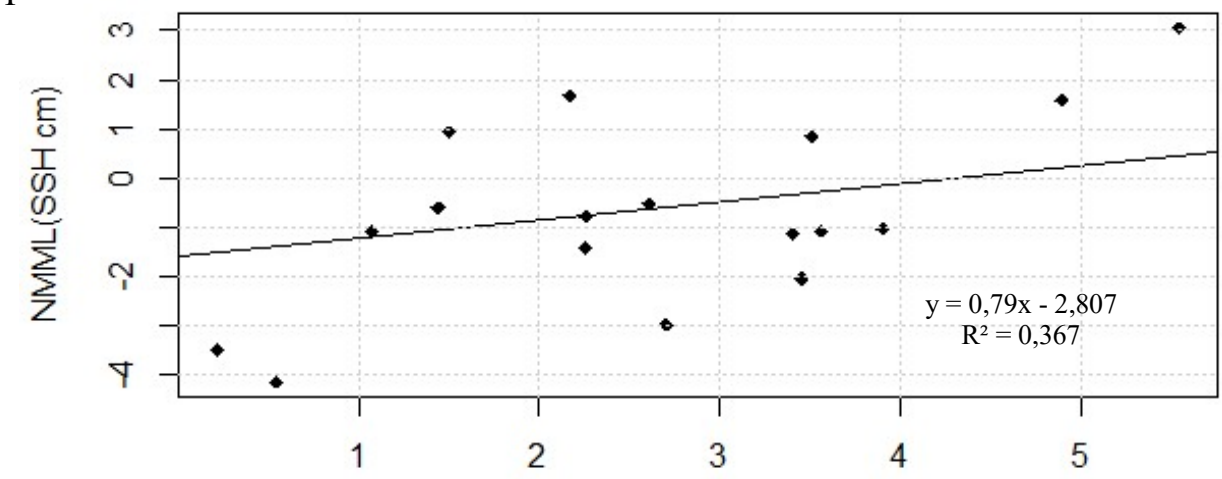

$\operatorname{NMMG}(\mathrm{cm})$

Fuente: Elaboración propia.

Las predicciones de altura del nivel en Valencia para los escenarios RCP2.6, RCP4.5 y RCP8.5, calculados a partir de los registros del mareógrafo del puerto, presentan alturas entre 797 y $853 \mathrm{~cm}$ para el periodo 2080-2100 (Figura 7); en los datos aportados por los satélites altimétricos la altura del nivel del mar se sitúa entre 29 y $47 \mathrm{~cm}$ (Figura 8). 
Figura 7. Expectativas de los niveles medios del mar mediante el mareógrafo de Valencia para

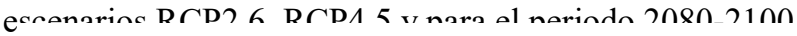

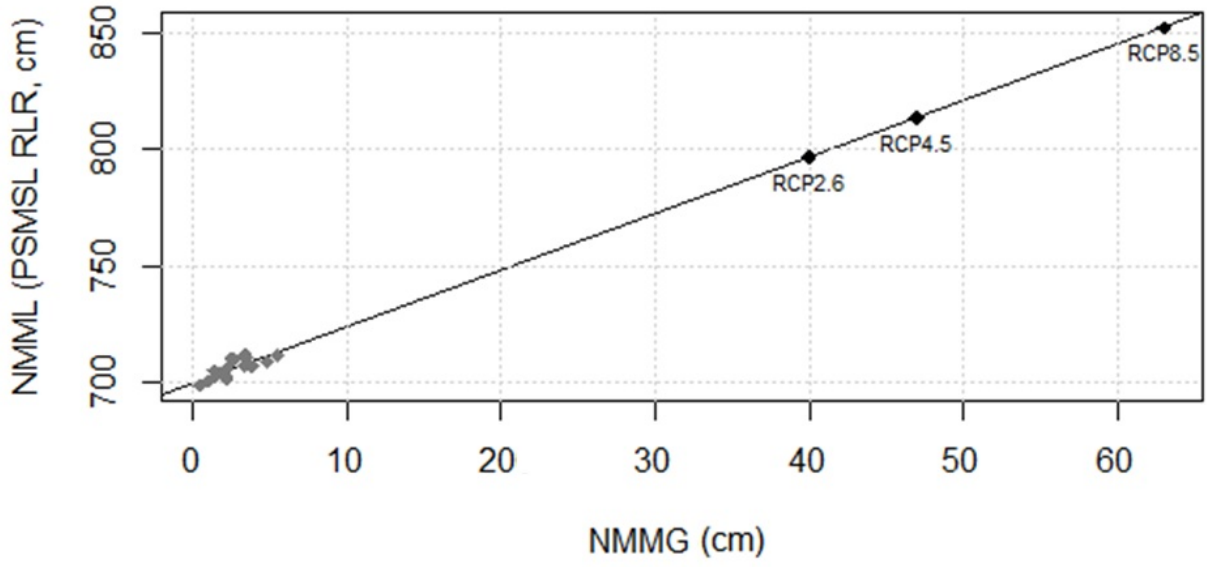

Fuente: Elaboración propia.

Figura 8. Predicción de los niveles medios del mar mediante satélites altimétricos de los tres

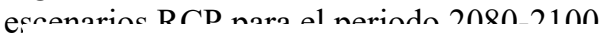

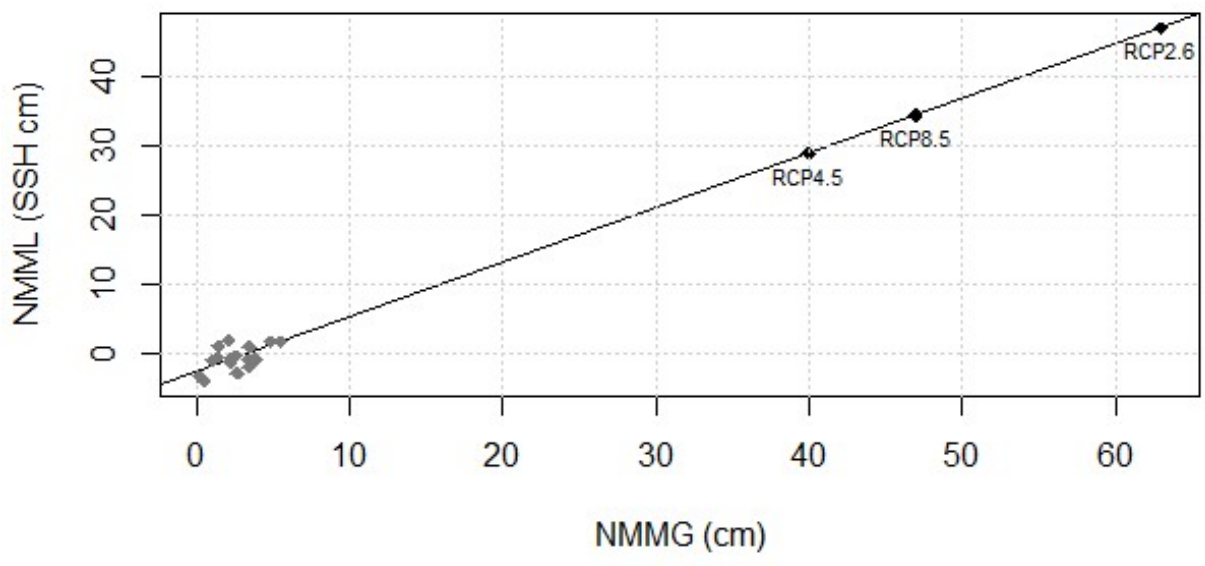

Fuente: Elaboración propia.

Los valores obtenidos en los modelos han servido para calcular los incrementos del nivel del mar, reflejados en la Tabla 3. En ella se muestran las expectativas locales del cambio del nivel del mar en la costa de Valencia para el periodo 2080-2100, mediante las dos fuentes de datos (mareógrafos y satélites). Las expectativas analizadas mediante el mareógrafo del puerto de Valencia, señalan en los tres escenarios subidas por encima del aumento global del IPCC, incluso considerando el umbral de probabilidad más bajo (2,5\%), destaca el escenario RCP8.5 de la ciudad de 
Valencia, con una diferencia de incremento respecto a la tendencia global de $0,8 \mathrm{~m}$. En el umbral de probabilidad más alto $(97,5 \%)$ las subidas son mayores de un metro en los dos primeros escenarios (RCP2.6 y RCP4.5) y superior a un metro en el escenario más extremo (RCP8.5).

Tabla 3. Predicción puntual y por intervalo de confianza del $95 \%$ del incremento medio del nivel del mar. Periodo 2080-2100.

\section{Incremento (cm)}

\section{Escenario IPCC Datos de satélite Datos de mareógrafo}

\begin{tabular}{lccc}
\hline $\boldsymbol{R C P 2 . 6}$ & $40 \pm 28,42$ & $27,59 \pm 21,31$ & $87,69 \pm 40,25$ \\
$\boldsymbol{R C P 4 . 5}$ & $47 \pm 29,40$ & $33,12 \pm 25,30$ & $104,71 \pm 47,82$ \\
$\boldsymbol{R C P 8 . 5}$ & $63 \pm 37,24$ & $45,76 \pm 34,42$ & $143,63 \pm 65,13$ \\
\hline
\end{tabular}

Fuente: Elaboración propia.

En cuanto a los registros procedentes de los satélites altimétricos, los valores de subida del nivel del mar no llegan a alcanzar los 0,5 m, independientemente del RCP tomado. Además, a diferencia de los registros anteriores solo se observan subidas por encima de los escenarios del IPCC en el intervalo de confianza del 97,5\%. Este comportamiento se puede explicar por la disparidad de estas dos fuentes de datos, tanto la de los mareógrafos como la del satélite altimétrico. Los satélites altimétricos, a pesar de obtener datos de toda la superficie oceánica, no tiene en cuenta la subsidencia, como los mareógrafos, llegando a dificultar la realización de estudios más ajustados a la realidad (Fraile Jurado et al., 2014).

Por otro lado, la estimación de subida del nivel del mar mediante modelos estadísticos conlleva errores estimados. El IPCC ha calculado para los tres escenarios el error asociado al nivel de confianza del 95\% (IPCC, 2013); estos errores son más reducidos respecto los márgenes de confianza obtenidos para la ciudad de Valencia mediante datos procedentes del puerto, y más altos respecto a los registros derivados de los satélites altimétricos para la celdilla de selección aleatoria de $1^{\circ} \times 1^{\circ}$ de Valencia (Tabla 4).

Aunque en el Mediterráneo Occidental existen pocos trabajos que estudian este tema en detalle, los resultados obtenidos son semejantes a los niveles estimados en ellos. Así por ejemplo, en la Tesis Doctoral realizada en la costa de la Región de Murcia (Ibarra Marinas, 2016), aunque solo se tiene en cuenta datos de satélites altimétricos, descartando datos de mareógrafos cercanos con series periódica completas, se obtienen valores similares, con pequeñas diferencias de 0,1-0,6 m, según el escenario RCP utilizado. 
Tabla 4. Error en predicción asociado al nivel de confianza del 95\% en cada uno de los escenarios en el periodo 2081-2100.

Error Valencia (cm)

\begin{tabular}{cccc}
\hline Escenario & IPCC & Satélite & Mareógrafo \\
\hline $\boldsymbol{R C P 2 . 6}$ & 14,5 & 10,87 & 20,54 \\
$\boldsymbol{R} \boldsymbol{C P} 4.5$ & 15 & 12,91 & 24,4 \\
$\boldsymbol{R C P 8 . 5}$ & 19 & 17,56 & 33,23 \\
\hline
\end{tabular}

Fuente: Elaboración propia.

Fraile Jurado et al. (2014) estiman el comportamiento futuro del nivel del mar en las Islas Canarias a partir de una metodología similar a la utilizada en este estudio, caracterizando las predicciones en esta zona del Océano Atlántico mediante datos altimétricos de satélite y de mareógrafos. Obtienen resultados medios no muy dispares con los obtenidos en este estudio. Sin embargo, si se tienen en cuenta las predicciones en el rango de confianza utilizado, las diferencias estimadas superan el medio metro en el escenario RCP8.5.; éste es el caso del modelo de Santa Cruz de Tenerife, que muestra subidas de 1,54 m en el escenario RCP8.5 (97,5\%), mientras que en Valencia la subida del nivel del mar para este mismo escenario y este mismo umbral de confianza es de 2,09 $\mathrm{m}$. Esta disparidad es debido al efecto de factores de compleja interpretabilidad en los registros de los mareógrafos, que reflejan rasgos marinos y terrestres, como los movimientos verticales relacionados con la tectónica local y regional (Fraile Jurado et al., 2014). Estas diferencias puede ser uno de los aspectos más discutibles de la metodología utilizada. Sin embargo, al analizar los resultados obtenidos con los datos de los satélites altimétricos, se observan diferencias mínimas con algunas de las celdillas del estudio realizado en las Islas Canarias.

Más recientemente, Sainz de Murieta et al. (2018) realizan un estudio similar en el País Vasco mediante datos de subida del nivel del mar estimados a partir del estudio de Kopp et al. (2014) y datos de mareógrafos. Las expectativas de incremento son inferiores a las obtenidas en este estudio para la costa valenciana, llegando incluso a duplicar los valores obtenidos en los diferentes escenarios RCP para el horizonte 100. Estas diferencias pueden explicarse por la variabilidad espacial o local, y a la metodología empleada: mediante el modelo de series de tiempo Browniano Geométrico (GBM) y la Distribución del Valor Extremo (GEV) (Sainz de Murieta et al., 2018).

Las estimaciones del nivel del mar son muy complejas y presentan incertidumbres derivadas de los numerosos factores a considerar (IPCC, 2013), por lo que no se puede saber con seguridad el escenario futuro de emisiones de efecto invernadero; es la denominada incertidumbre de escenario (ACE CRC, 2008), derivada de un futuro 
social, económico y de desarrollo ambiental del mundo incierto. Sin embargo, a pesar de las incertidumbres existentes, todas las simulaciones apuntan a un incremento del nivel del mar para los siguientes 100 años (Church et al., 2001; Church y White, 2011; IPCC, 2013). De los tres escenarios futuros, el escenario RCP4.5 y, sobre todo, el RCP8.5 (asociado a altas emisiones) presentan subidas del nivel del mar mayores. Si se analiza la tendencia actual de los indicadores de cambio climático, y se tiene en cuenta el grado de cumplimiento de los acuerdos al alcanzar, es plausible considerar ambos escenarios como comportamiento futuro. Además, tal y como señalan Mengel et al. (2018), incluso si se llevan a cabo todas las medidas de mitigación propuestas en la cumbre de Paris, éstas serán insuficientes, estimando en su modelos de pronóstico subidas entre 0,7 y $1,2 \mathrm{~m}$ en el caso de emisiones de gases de efecto invernadero netas nulas.

\section{Conclusiones}

Debido a su carácter cambiante, el litoral constituye uno de los medios más sensibles al Cambio Climático. El papel de la Geografía resulta imprescindible para realizar proyecciones a largo plazo. Las Ciencias Espaciales permiten la obtención de datos de aproximación a los fenómenos y el acceso a los mecanismos que causan sus efectos y facilitan la toma de decisiones destinadas a la ordenación del territorio.

La variabilidad de la subida del nivel del mar y las diferencias de sus efectos en áreas concretas, hacen necesarios los estudios de carácter regional que tengan en cuenta tanto los cambios de nivel a escala local, como las características geográficas de la costa.

El efecto del ascenso sobre la erosión de las playas se verá agravado por los temporales y por la reducción del aporte de sedimentos, debido a los cambios en el régimen hídrico, por efecto del Cambio Climático.

El efecto de un aumento del nivel del mar de 143,63cm, en el caso de los mareógrafos para el escenario RCP8.5, puede parecer escasa en las playas con perfiles reflectantes, pero es trascendental tener en cuenta que el ascenso del nivel del mar amplifica el efecto de los temporales, este hecho representa el principal problema del efecto del Cambio Climático en la costa.

El estudio del efecto de la subida del nivel del mar está sujeto a diferentes parámetros, que dificultan la exactitud de las proyecciones, ya que los propios cambios implican nuevos reajustes de la costa. La utilización de los modelos de elevaciones actuales, no tienen en cuenta los cambios en la topografía a largo plazo. Sin embargo, la aportación de estas herramientas espaciales es esencial para establecer aproximaciones de los efectos de este y otros procesos marinos. 


\section{Referencias bibliográficas}

ACE CRC (2008): Position analysis: climate change, sea-level rise and extreme events: impacts and adaptation issues. Antarctic Climate \& Ecosystems Cooperative Research Centre.

Alvarado-Aguilar D., Jiménez J.A. y Nicholls R.J. (2012): Flood Hazard and damage assessment in the Ebro Delta (NW Mediterranean) to relative sea level rise. Natural Hazards, 62, 1301-1321. DOI 10.1007/s11069-012-0149-x

Álvarez Francoso J.I., Fraile Jurado, P. y Ojeda Zújar J. (2017): Cartografía de la probabilidad de inundación como consecuencia de la subida del nivel medio del mar en Punta Umbría, Huelva. En Geo-Temas. IX Jornadas de Geomorfología Litoral. Menorca, Sociedad Geológica de España, 1576-5172.

Arteaga Cardineau, C. (2007-2008): Aproximación a la erosión costera en la Isla de Olerán (Charente Maritime, Francia). Territoris, 7, 115-133.

Benavente, J., Del Río, L., Gracia, F.J. y Martínez del Pozo, J.A. (2006): Coastal flooding hazard related to storms and coastal evolution in Valdelagrana spit (Cadiz Bay Natural Park, SW Spain). Continental Shelf Research, 26, 1061-1076. DOI 10.1016/j.csr.2005.12.015

Bond G., Showers W., Elliot M., Evans M., Lotti R., Hadjas I., Bonani G. y Johnson S. (2013): The North Atlantic's 1-2 kyr climate rhythm: Relation to Heinrich events Dansgaard/Oeschger Cycles and the Little Ice Age. En Clark, U., Webb, S.y Keigwin, D. (Eds): Mechanisms of global climate change at millennial time scales. Washington, D. C., American Geophysical Union Monograph, 35-58. DOI 10.1029/GM112p0035

Brown, J.M., Ciavola, P., Masselink, G., McCall, R.y Plater, A.J. (2016): Monitoring and modelling to guide coastal adaptation to extreme storm events in a changing climate. Natural Hazards Earth System Science, 16, 463-467. DOI10.5194/nhess-16-463-2016

Bruun P. (1962): Sea-level rise as a cause of soil erosion. American Society of Civil Engineering Proceedings, Journal of Waterways and Harbours Division, 88, 117-130.

Cazenave, A. y Llowell, W. (2010): Contemporary Sea Level Rise. Annual Review of Marine Science, 2,145-173. DOI 10.1146/annurev-marine-120308-081105

Chapman M.R. y Shackleton, N.J. (2000): Evidence of 550-year and 1000 year cyclities in North Atlantic circulat on patterns during the Holocene. The Holocene, 10, 287-291. DOI 10.1139/E08-037

Church, J., Gregory, J., Huybrechts, P., Kuhn, M. Lambeck, K., Nhuan, M., Qin, D. y Woodworth, P. (2001): Changes in sea level, in Climate Change 2001: The Scientific Basis. En Houghton, J.T., Ding, Y., Griggs, D.J., Noguer, M., van der Winden, P.J. y Dai, X. (Eds): Contribution of Working Group I to the Third Assessment Report of the Intergovernmental Panel on Climate Change, edited by J. Houghton et al., chap. 11, pp.639-694.

Church, J.A. y White, N.J. (2011): Sea-level rise from the late 19th to the early 21 st century. Surveys in Geophysics, 32(4-5): 585-602. DOI 10.1007/s10712-011-9119-1

Clarke, L.E., Edmonds, J.A., Jacoby, H.D., Pitcher, H.M., Reilly, J.M. y Richels, R.G. (2007): Scenarios of Greenhouse Gas Emissions and Atmospheric Concentrations. Sub report 2.1A of Synthesis and Assessment Product 2.1 by the U.S. Climate Change Science Program 
and the Subcommittee on Global Change Research. Department of Energy, Office of Biological \& Environmental Research.

Dangendorf, S., Marcos, M., Wöppelmann, G., Conrad, C.P., Frederikse, T. y Riva, R. (2017): Reassessment of 20thcentury global mean sea level rise. Procedings of the National Academy of Sciences of the US, 114(23), 5946-5951.

Del Rio, L., Benavente, J., García, F.J. y Chica, J.A. (2015): Riesgos de erosión en la costa de Cádiz: gestión actual y perspectivas futuras. Geo-Temas, 15, 149-152.

Eguibar Galán, M.A., Sanchis Ibor, C., Marco Segura J.B. yMateuBellés, J.F. (2007): El Catálogo de Zonas Humedas de la Comunidad Valenciana. Aspectos metodológicos. Ingeniería del Agua, 14(1), 23-35. DOI 10.4995/ia.2007.2900

Fraile Jurado, P. (2011): Análisis de las problemáticas asociadas a la espacialización, evolución y representación de niveles del mar presentes y Futuros en Andalucía. (Tesis doctoral). Universidad de Sevilla.

Fraile Jurado, P. y Ojeda Zujar, P. (2012): The importance of the vertical accuracy of digital elevation models in gauging inundation by sea level rise along the Valdelagrana beach and marshes (Bay of Cádiz, SW Spain). Geo-Marine Letters, 33(2), 225-230. DOI 10.1007/s00367-012-0317-8

Fraile Jurado, P., Sánchez Rodríguez, E., Fernández Díaz, M. y Pita López, M.F. (2014): Estimación del comportamiento futuro del nivel del mar en las Islas Canarias a partir del análisis de registros recientes. Geographicalia, 66, 79-98.DOI 10.26754/ojs_geoph/geoph.2014661066

García Fernández, C. (2010): La evidencia del cambio climático. La necesidad de las políticas económicas preventivas. Nómadas, Revista Crítica de Ciencias Sociales y Jurídica, 25, 4766.

Gomis, D., Ruiz, S., Sotillo, M.G., Álvarez-Fanjul, E. y Terradas J. (2008): Low frequency Mediterranean sea level variability: The contribution of atmospheric pressure and wind. Global and Planetary Change, 63, 215-229. DOI 10.1016/j.gloplacha.2008.06.005

Gonzalez M. y Medina, R. (2001): On the application of static equilibrium bay formulations to natural and man-made beaches. Coastal Engineering, 43, 3-4, 209-225. DOI 10.1016/S0378-3839(01)00014-X

Han, G., Ma, Z., Bao, H. y Slangen, A. (2014): Regional differences of relative sea level changes in the Northwest Atlantic: Historical trends and future projections. Journal of Geophysical Research, 119(1), 156-164.

Hidalgo M.C. y Picaso I. (2010): Predictores de la percepción de riesgo y del comportamiento ante el cambio climático. Un estudio piloto. Psyecology, 1, 39-46. DOI $10.1174 / 217119710790709577$

Hinkel, J., Lincke, D., Vafeidis, A.T., Perrette, M., Nicholls, R..J., Tol, R.S. J., Marzeion, B., Fettweis, X., Ionescu, C. y Levermann, A. (2014): Coastal flood damage and adaptation costs under 21st century sea-level rise. PNAS, National Academy of Sciences, 111(9), 3292-3297. DOI 10.1073/pnas.1222469111

Holgate, S.J., Matthews A., Woodworth, P.L., Rickards, L.J., Tamisiea, M.E., Bradshaw, E., Foden, P.R., Gordon, K.M., Jevrejeva S. y Pugh, J. (2013): New Data Systems and 
Products at the Permanent Service for Mean Sea Level. Journal of Coastal Research, 29(3), 493-504.DOI10.2112/JCOASTRES-D-12-00175.1

Ibáñez, C., Prat, N. y Canicio, A. (1996): Changes in the hydrology and sediment transport produced by large dams on the lower Ebro River and its estuary. River Research and Applications, 12, 51-62. DOI 10.1002/(SICI)1099-1646(199601)12:1<51::AIDRRR376>3.0.CO;2-I

Ibarra Marinas, A.D., Belmonte Serrato, F., Gomariz Castillo, F. y Pérez Cutillas, P. (2015): Evolución de la línea de costa en la Región de Murcia (1956-2013). Geo-Temas, 15, 33-36. Ibarra Marinas, A.D., (2016): Análisis y Evolución de las Playas de la Región de Murcia (1956-2013). Tesis Doctoral. Universidad de Murcia.

Instituto Geográfico Nacional (IGN) (2017): Cartociudad: proyecto colaborativo de producción y publicación del Sistema Cartográfico Nacional. Disponible en internet: http://www.cartociudad.es/portal/

IPCC (2007): Climate Change 2007 Impacts adaptation and vulnerability. Cambridge University Press.

IPCC (2013): Climate Change 2013: The Physical Science Basis. Contribution of Working Group I to the Fifth Assessment Report of the Intergovernmental Panel on Climate Change. Stocker, T.F., Qin, D., Plattner, G.K., Tignor, M., Allen, S.K., Boschung, J., Nauels, A., Xia, Y., Bex, V. y Midgley, P.M. (Eds.). Cambridge University Press, Cambridge.

IPCC (2014): Climate change 2014: Synthesis report Contribution of working groups I, II and III to the fifth assessment report of the intergovernmental panel on climate change.Pachauri, R.K. yMeyer, L.A. (Eds.). World Meteorological Organization, Geneva, Suiza.

IPCC (2016): Climate Change 2013: The Physical Science Basis. Working Group I Contribution to the Fifth Assessment Report of the Intergovernmental Panel on Climate Change. Stocker TF, Qin D, Plattner G-K, et al. (Eds).Cambridge University Press, Cambridge.

Jevrejeva, S., Moore, J. C. y Grinstead, A. (2012): Sea level projections to AD2500 with a new generation of climate change scenarios.Global and Planetary Change, 80-81, 14-20. DOI 10.1016/j.gloplacha.2011.09.006

Jevrejeva, S., Jackson, L.P., Riva, R. Grinsted, A. y Moore, J. (2016): Coastal sea level rise with warming above $2^{\circ} \mathrm{C}$. Proceedings of the National Academy of Sciences, 113(47), 13342-13347.DOI 10.1073/pnas.1605312113

Kopp, R.E., Horton, R.M., Little, C.M., Mitrovica, J.X., Oppenheimer, M., Rasmussen, D.J., Strauss, B.H. y Tebaldi, C. (2014): Probabilistic 21st and 22nd century sea-level projections at a global network of tide-gauge sites. Earth'sFuture, 2, 383-406. DOI 10.1002/2014EF000239

Lizano, A. y Lizano, O. (2010): Creación de escenarios de inundación en la Ciudad de Puntarenas ante el aumento del nivel del mar. Intersedes, 11, 215-229.

Loutre M.F., Berger, A., Bretagnon P. y Blanc, P.L. (1992): Astronomical frequencies for climate research at the decadal to century time scale. Climate Dynamics, 7, 181-194. DOI 10.1007/BF00206860

Marine Information Service (2016): EMODnet Digital Bathymetry (DTM). Marine Information Service. DOI 10.12770/c7b53704-999d-4721-b1a3-04ec60c87238 
Meinshausen, M., Smith, S.J., Calvin, K.V., Daniel, J.S., Mikiko, K., Lamarque, J.F., Ken'ichi, M., Montzka, S.A., Raper, S.C.B., Keywan, R., Thomson, A.M., Velders, G.J.M. y Detlef, V. (2011): The RCP greenhouse gas concentrations and their extensions from 1765 to 2300 . ClimaticChange, 109(1-2), 213-241. DOI 10.1007/s10584-011-0156-Z

Mengel, M., Nauels, A., Rogelj, J. y Schleussner, C.F. (2018): Committed sea-level rise under the Paris Agreement and the legacy of delayed mitigation action. Nature Communications, 9(1), 601. DOI 10.1038/s41467-018-02985-8

Ministerio de Agricultura y Pesca, Alimentación y Medio Ambiente (MAPAMA) (2017): Ecocartografías deValencia. Disponiblesen internet: http://www.mapama.gob.es/es/costas/temas/proteccion costa/ecocartografias/ecocartografia-valencia.aspx

Nerem, R. S., Beckley, B. D., Fasullo, J. T., Hamlington, B. Masters, D. y Mitchum G. T. (2018): Climate-change-driven accelerated sea-level rise detected in the altimeter era. Proceedings of the National Academy of Sciences. DOI 10.1073/pnas.1717312115

Nerem, R.S., Chambers, D.P., Leuliette, E.W., Mitchum, G.T. y Giese, B.S.(1999): Variations in global mean sea level associated with the 1997-1998 ENSO event: Implications for measuring long-term sea level change. Geophysical Research Letters, 26(19), 3005-3008. DOI 10.1029/1999GL002311

Ojeda Zújar J., Álvarez Francoso, J.I., Martín Cajaraville D. y Fraile Jurado P. (2009): El uso de las TIG para el cálculo del índice de vulnerabilidad costera (CVI) ante una potencial subida del nivel del mar en la costa andaluza (España). GeoFocus, 9, 83-100.

Pernía Llera JM. y Fornés Azcoiti JM., (2008): Cambio climático y Agua subterráneas: Visión para los próximos decenios. Madrid. Instituto Geológico y Minero de España.

Pitarch Garrido, M.D. y Membrado Tena, J.C. (2014): Análisis de la evolución del suelo urbano durante la burbuja inmobiliaria (1996-2006) en las principales ciudades valencianas. Cuadernos de Geografía, 95-96, 149-173.

Pons Valls, J.M. (2009): La medición del nivel medio del mar. Mapping, 135, 6-10.

Puertos del Estado (2017): Datos de oceanografía y SIMAR. Disponibles en internet: http://www.puertos.es

QGIS Development Team (2017): QGIS Geographic Information System. Open Source Geospatial Foundation Project. Disponible en internet: http://qgis.osgeo.org

R Core Team (2017). R: A language and environment for statisticalcomputing. R Foundation for Statistical Computing, Vienna, Austria. Disponibleen internet: https://www.Rproject.org/.

Rahmstorf, S., (2007): A semi-empirical approach to projecting future sea-level rise. Science, 315, 368-370. DOI 10.1126/science.1135456

Riahi, K., Gruebler, A. y Nakicenovic N. (2007): Scenarios of long-term socioeconomic and environmental development under climate stabilization. TechnologicalForecasting and Social Change, 74(7), 887-935. DOI 10.1016/j.techfore.2006.05.026

Sainz de Murieta, E., Abadie, L.M. y Galarraga I. (2018): Aumento del nivel del mar en la costa vasca: una aproximación probabilística. Ekonomiaz, 94 (2), 324-344. 
Sanjaume E., Rossello V.M., Pardo J.E., Carmona P., Segura, F. y López Garcia, M.J. (1996): Recent Coastal Changes in the Gulf of Valnecia (Spain). Zeitchfür Geomorphologie, 102, 95-118.

Schubert, R., Schellnhuber, H.J., Buchmann, N., Epiney, E., Griebhammer, R., Kulessa, M. y Schmid, J. (2006): The future oceans-Warming up, rising high, turning sour, Berlín, German Advisory Council on Global Change (WBGU).

Spencer, N.E. y Woodworth, P.L. (1993): Data holdings of the Permanent Service for Mean Sea Level (November 1993). Bidston, Birkenhead: PermanentServicefor Mean Sea Level.

Tavares Correa, C. y Drenkhan, F. (2010): Variación de la línea de litoral entre puerto Chicama y Puémape, La Libertad, Perú (1961-2006). Espacio y Desarrollo, 22, 103-117.

Van Vuuren, D., den Elzen, M., Lucas, P., Eickhout, B., Strengers, B., van Ruijven, B.,Wonink, S. y van Houdt, R. (2007): Stabilizing greenhouse gas concentrations at low levels: an assessment of reduction strategies and costs. Climatic Change, 81, 119-159. DOI 10.1007/s10584-006-9172-9

Vaughan, D.J. (2006): Recent trends in melting conditions on the Antarctic Peninsula and their implications for ice sheet mass balance and sea level Arctic. Antarctic and Alpine Research, 38(1): 147-152. DOI 10.1657/1523-0430(2006)038[0147: RTIMCO] 2.0.CO; 2

Williams, S.J. (2013): Sea Level Rise Implications for Coastal Regions. Journal of Coastal Research, 63, 184-196. DOI 10.2112/SI63-015.1

Wise, M.A., Calvin, K.V., Thomson, A.M., Clarke, L.E., Bond-Lamberty, B., Sands, R.D., Smith, S.J., Janetos, A.C. y Edmonds, J.A. (2009): Implications of limiting CO2 concentrations for land use and energy. Science, 324, 1183-1186.

Yi, S., Sun, W. y Heki, K. (2016): An increase in the rate of global mean sea level rise since 2010. Geophysical Research Letters, 42(10), 3998-4006. DOI 10.1002/2015GL063902 